\title{
Interaction due to Hall Current and Rotation in a Modified Couple Stress Elastic Half-Space due to Ramp-type Loading
}

\author{
Rajneesh Kumar $^{1 *}$, Shaloo Devi ${ }^{2}$ \\ ${ }^{1}$ Department of Mathematics, Kurukshetra University Kurukshetra \\ Kurukshetra, India \\ ${ }^{*}$ E-mail: rajneesh_kuk@rediffmail.com \\ ${ }^{2}$ Department of Mathematics \& Statistics, Himachal Pradesh University Shimla \\ Shimla, India \\ E-mail: shaloosharma2673@gmail.com
}

Received: 29 April 2015; revised: 21 December 2015; accepted: 21 December 2015; published online: 29 December 2015

\begin{abstract}
The present investigation is to focus on the effect of Hall current and rotation in a modified couple stress theory of elastic half space due to ramp-type loading in a homogeneous, isotropic, thermoelastic diffusive medium. The mathematical formulation is prepared for different theories of thermoelastic diffusion, including the Coriolis and centrifugal forces. The Laplace and Fourier transforms techniques are applied to obtain the solutions of the governing equations. The components of displacement, stresses, temperature change and mass concentration are obtained in the transformed domain. The numerical inversion technique has been used to obtain the solutions in the physical domain. Effects of Hall current and rotation are shown on the resulting quantities. Some particular cases are also discussed in the present problem.

Key words: modified couple stress, generalized thermoelasticity, Laplace and Fourier transforms, ramp-type Loading, Hall current and rotation
\end{abstract}

\section{INTRODUCTION}

Couple-stress theory is an extended continuum theory that includes the effects of a couple per unit area on a material volume, in addition to the classical direct and shear forces per unit area. This immediately admits the possibility of a symmetric stress tensor, since shear stress no longer has to be conjugate in order to ensure rotational equilibrium. Some researchers investigated some problems on the couple stress theory which were not paid enough attention for a long time comparing with classical elasticity.

The classical couple stress theory, originated by Toupin [1], Mindlin and Tiersten [2] and Koiter [3], is one of the nonclassical theories containing two higher-order material length scale parameters in addition to the two classical Lame constants for isotropic elastic materials in its constitutive equation. Zhao et al. [4] discussed the problem of weak con- tinuity condition of FEM for the axisymmetric couple stress theory and an 18-DOF triangular axisymmetric element. Also, Reddy [5] studied microstructure-dependent couple stress theories of functionally graded beams. Recently, the boundary element formulation for plane problems in couple stress elasticity discussed by Hadjesfandiari and Dargush, [6].

Yang et al. [7] introduced the modified couple stress theory. Beside the two conventional equilibrium relationships in the classical couple stress, they proposed an additional relation to constrain the couple. This relation considers the balance of moment of rotational momentum. This assumption make the couple stress tensor symmetric.Recently the couple stress theory has attracted much attention because it describes the size effect of materials, whose theoretical and numerical method of analysis becomes a new research spot (e.g., Shankar et al. [8], Babaoglu and Erbay [9], Diebels S, Steeb H. [10] and Kulesh et al. [11]). 
Park and Gao [12] developed an Euler-Bernoulli beam model based on the modified couple stress theory and uniaxial stress-strain relationships. Ma et al. [13] developed Euler-Bernoulli and Timoshenko beam models based on the modified couple stress theory and three-dimensional stressstrain relationships. Marin [14] discussed the problem of dipolar materials with stretch. Tsiatas [15] studied the static bending analysis of isotropic micro-Kirchhoff plates using the modified couple-stress theory and on the basis of the principle of minimum potential energy.

Ma et al. [16] developed the Mindlin plate model using the modified couple-stress theory and on the basis of Hamilton's principle. Asghari [17] studied the geometrically nonlinear micro-plate formulation based on the modified couple stress theory. Marin and Gabrial [18] studied weak solutions in elasticity of dipolar bodies with stretch. Simsek and Reddy [19] investigated the bending and vibration of fuctionally graded microbeams using a new higher order beam theory and the modified couple stress theory. Marin et al. [20] investigated the problem of nonsimple material problems addressed by the Lagrange's identity. Recently, the size dependent buckling analysis of microbeams based on themodified couple stresss theory with high order theories and general boundary conditions have been studied by Mohammad-Abadi and Daneshmehr [21]. Shaat et al. [22] studied the size-dependent bending analysis of Kirchhoff nano-plates based on the modified couple-stress theory including surface effects. Ghorbanpour Arani et al. [23] discussed the problem of vibration of bioliquid-filled microtubules embedded in cytoplasm including surface effects using the modified couple stress theory. Recently, nonlinear bending and post-buckling of extensible microscalebeams based on the modified couple stress theory have been studied by Wang et al. [24].

Thermodiffusion in an elastic solid is due to the coupling of the fields of temperature, mass diffusion and strain. Heat and mass exchange with the environment during the process of thermodiffusion in an elastic solid. The concept of thermodiffusion is used to describe the processes of thermomechanical treatment of metals (carboning, nitriding steel, etc.) and these processes are thermally activated, and their diffusing substances include nitrogen, carbon, etc. They are accompanied by deformations of the solid. Podstrigach [25] considered the problem of thermodiffustion in classical elastic material and investigated the fundamental corollaries and differential equations. Nowacki [26-29] developed the theory of thermoelastic diffusion by using coupled thermoelastic model. Sherief et al. [30] developed the theory of generalized thermoelastic diffusion that predicts finite speeds of propagation for thermoelastic and diffusive waves. Sherief and Saleh [31] worked on a problem of thermoelastic half space with a permeating substance in contact with the bounding plane in the context of the theory of generalized thermoelastic diffusion with one relaxation time. Recently, Kumar and Kansal [32] derived the basic equations in generalized thermoelastic diffusion for Green Lindsay (GL-model) theory and discussed the Lamb waves.

The foundations of magnetoelasticity were presented by Knopoff [33] and Chadwick [34] and developed by Kaliski and Petykiewicz [35]. An increasing attention is being devoted to the interaction between magnetic field and strain field in a thermoelastic solid due to its many applications in the fields of geophysics, plasma physics and related topics. In all papers quoted above it was assumed that the interactions between the two fields take place by means of the Lorentz forces appearing in the equations of motion and by means of a term entering Ohm's law and describing the electric field produced by the velocity of a material particle moving in a magnetic field.

When the magnetic field is very strong, the conductivity will be a tensor and the effect of Hall current cannot be neglected. The conductivity normal to the magnetic field is reduced due to the free spiraling of electrons and ions about the magnetic lines of force before suffering collisions, and a current is induced in a direction normal to both the electric and magnetic fields. This phenomenon is called the Hall effect. In all of the above investigations, the effects of Hall current have not been considered. Effects of Hall current and rotation on magneto-microploar generalized thermoelasticity due to ramp-type heating was studied by Zakaria [36]. Zakaria [37] also investigated the effect of Hall current on generalized magneto-thermoelasticity micropolar solid subjected to ramp-type heating.

The present investigation is to determine the components of displacement, stresses, temperature change and mass concentration in a modified couple stress generalized thermoelastic with mass diffusion under the influence of Hall current and rotation by applying Laplace and Fourier transforms. The ramp-type loading is applied on the mechanical boundaries to get the solution in the complete form. Some particular cases are also derived from the present investigation.

\section{BASIC EQUATIONS}

Following [7, 32, 36], the field equations in a modified couple stress thermoelastic medium with mass diffusion in the absence of body forces, body couples, heat and mass diffusion sources are given by:

(i) Constitutive relations

$$
\begin{gathered}
t_{i j}=\lambda e_{k k} \delta_{i j}+2 \mu e_{i j}-\frac{1}{2} e_{k i j} m_{l k, l} \\
-\beta_{1}\left(1+\tau_{1} \frac{\partial}{\partial t}\right) T \delta_{i j}-\beta_{2}\left(1+\tau^{1} \frac{\partial}{\partial t}\right) C \delta_{i j}, \\
m_{i j}=2 \alpha \chi_{i j}, \\
\chi_{i j}=\frac{1}{2}\left(w_{i, j}+w_{j, i}\right),
\end{gathered}
$$




$$
w_{i}=\frac{1}{2} e_{i p q} u_{q, p},
$$

(ii) Equations of motion in the rotation frame of reference are

$$
\begin{aligned}
& \left(\lambda+\mu+\frac{\alpha}{4} \Delta\right) \nabla(\nabla \cdot \mathbf{u})+\left(\mu-\frac{\alpha}{4} \Delta\right) \nabla^{2} \mathbf{u} \\
- & \beta_{1}\left(1+\tau_{1} \frac{\partial}{\partial t}\right) \nabla T-\beta_{2}\left(1+\tau^{1} \frac{\partial}{\partial t}\right) \nabla C+\mathbf{F}= \\
= & \rho(\ddot{\mathbf{u}}+\boldsymbol{\Omega} \times(\boldsymbol{\Omega} \times \mathbf{u})+2(\boldsymbol{\Omega} \times \dot{\mathbf{u}})),
\end{aligned}
$$

(iii) Equation of heat conduction

$$
\begin{gathered}
K^{*} \triangle T-\rho c_{e}\left(\frac{\partial}{\partial t}+\tau_{0} \frac{\partial^{2}}{\partial t^{2}}\right) T \\
-a T_{0}\left(\frac{\partial}{\partial t}+\gamma \frac{\partial^{2}}{\partial t^{2}}\right) C=T_{0} \beta_{1}\left(\frac{\partial}{\partial t}+\tau_{0} \eta_{0} \frac{\partial^{2}}{\partial t^{2}}\right)(\nabla \cdot \mathbf{u}),
\end{gathered}
$$

(iv) Equation of mass diffusion

$$
\begin{aligned}
& D \beta_{2} \Delta(\nabla \cdot \mathbf{u})+D a\left(1+\tau_{1} \frac{\partial}{\partial t}\right) \triangle T \\
+ & \left(\frac{\partial}{\partial t}+\tau^{0} \eta_{0} \frac{\partial^{2}}{\partial t^{2}}\right) C-D b \Delta\left(1+\tau^{1} \frac{\partial}{\partial t}\right) C=0 .
\end{aligned}
$$

Here, the medium is rotating with angular velocity, where $\boldsymbol{\Omega}=\Omega \widehat{\nu}$, where $\widehat{\nu}$ is the unit vector along the axis of rotation and these equations of motion include two additional terms, namely,

(i) The centripetal acceleration $\boldsymbol{\Omega} \times(\boldsymbol{\Omega} \times \mathbf{u})$ due to timevarying motion,

(ii) The Coriolis acceleration $2(\boldsymbol{\Omega} \times \dot{\mathbf{u}})$,

where $\lambda, \mu$ are material constants, $\alpha$ is the couple stress parameter and $\rho$ is the density, $\beta_{1}=(3 \lambda+2 \mu) \alpha_{t}, \beta_{2}=$ $(3 \lambda+2 \mu) \alpha_{c}$, Here $\alpha_{t}, \alpha_{c}$ are the coefficients of linear thermal expansion and diffusion expansion, respectively, $\mathbf{u}=\left(u_{1}, u_{2}, u_{3}\right)$ is the components of displacement vector, $T$ is the temperature change, $C$ is the mass concentration, $\Omega$ is the rotation, $K^{*}$ is the coefficient of thermal conductivity, $c_{e}$ is the specific heat at constant strain, $a$ is the coefficient describing the measure of thermoelastic diffusion, $T_{0}$ is the reference temperature assumed to be such that $\left|T / T_{0}\right| \ll 1, D$ is the thermoelastic diffusion constant, $b$ is the coefficient describing the measure of mass diffusion effects, $t_{i j}$ are the components of stress tensor, $m_{i j}$ are the components of couple-stress, $e_{i j}$ are the components of strain tensor, $\Delta$ is the Laplacian operator, $\nabla$ is del operator, $\chi_{i j}$ is symmetric curvature tensor, $\delta_{i j}$ is Kronecker's delta, $e_{i j k}$ is alternate tensor, $w_{i}$ is the rotational vector, Here $\tau^{0}, \tau^{1}$ are the diffusion relaxation times with $\tau^{1} \geq \tau^{0} \geq 0$ and $\tau_{0}, \tau_{1}$ are thermal relaxation times with $\tau_{1} \geq \tau_{0} \geq 0$. Here $\tau_{1}=\tau^{1}=0, \eta_{0}=1, \gamma=\tau_{0}$, for Lord-Shulman (L-S) model and $\eta_{0}=0, \gamma=\tau^{0}$, for Green Lindsay (G-L) model. Following Zakaria [36], the generalized Ohm's law including
Hall current:

$$
\mathbf{J}=\frac{\sigma_{0}}{1+m^{2}}\left[\mathbf{E}+\mu_{0}(\dot{\mathbf{u}} \times \mathbf{H})-\frac{\mu_{0}}{e n_{e}}(\mathbf{J} \times \mathbf{H})\right],
$$

and $\mathbf{F}=\mu_{0}(\mathbf{J} \times \mathbf{H})$ is the Lorentz force, where $\mathbf{J}$ is the current density vector, $\mu_{0}$ is the magnetic permeability, $\mathbf{H}$ is the total magnetic field vector, $\mathbf{E}$ is the intensity vector of the magnetic field, $m=\omega_{e} t_{e}$ is the Hall parameter, $t_{e}$ is the electron collision time, $\omega_{e}=e B_{0} / m_{e}$ is the electronic frequency, $e$ is the charge of an electron, $B_{0}$ is the magnetic induction, $m_{e}$ is the mass of the electron, $\sigma_{0}=e^{2} n_{e} t_{e} / m_{e}$ is the electrical conductivity and $n_{e}$ is the number density of electrons.

\section{FORMULATION AND SOLUTION OF THE PROBLEM}

A homogeneous isotropic, modified couple stress generalized thermoelastic elastic body with mass diffusion occupying the region of a half-space $x_{3} \geq 0$ is taken. We consider a rectangular Cartesian coordinate system $\left(x_{1}, x_{2}, x_{3}\right)$ having origin on the surface $x_{3}=0$. We consider a plane deformation problem with all the field quantities depending only on $\left(x_{1}, x_{3}, t\right)$. The half-surface is subjected to ramp-type loading on the bounding plane $x_{3}=0$ along with isothermal and isoconcentrated boundaries.

For two dimensional problem, we take

$$
\begin{gathered}
u_{i}=\left(u_{1}\left(x_{1}, x_{3}, t\right), 0, u_{3}\left(x_{1}, x_{3}, t\right)\right), \\
T\left(x_{1}, x_{3}, t\right), C\left(x_{1}, x_{3}, t\right) .
\end{gathered}
$$

Let us assume that the magnetic field $\mathbf{H}$ and the angular velocity $\Omega$ acts in the direction of $x_{2}$ axis as

$$
\begin{gathered}
\mathbf{H}=\left(0, H_{0}, 0\right), \\
\boldsymbol{\Omega}=(0, \Omega, 0) .
\end{gathered}
$$

We also assume that $E=0$, and the generalized Ohm's law $J_{2}=0$ everywhere in the medium.

With these considerations, the current density components $J_{1}$ and $J_{3}$ are given by

$$
\begin{aligned}
& J_{1}=\frac{\sigma_{0} B_{0}}{1+m^{2}}\left(m \frac{\partial u_{1}}{\partial t}-\frac{\partial u_{3}}{\partial t}\right), \\
& J_{3}=\frac{\sigma_{0} B_{0}}{1+m^{2}}\left(\frac{\partial u_{1}}{\partial t}+m \frac{\partial u_{3}}{\partial t}\right) .
\end{aligned}
$$


We define the dimensionless quantities:

$$
\begin{aligned}
& x_{i}^{\prime}=\frac{\omega^{*}}{c_{1}} x_{i}, \quad u_{i}^{\prime}=\frac{\omega^{*}}{c_{1}} u_{i}, t^{\prime}=\omega^{*} t, t_{i j}^{\prime}=\frac{t_{i j}}{\beta T_{0}}, \\
& m_{i j}^{\prime}=\frac{\omega^{*} m_{i j}}{c_{1} \beta T_{0}}, \gamma^{\prime}=\omega^{*} \gamma, \tau_{1}^{\prime}=\omega^{*} \tau_{1}, \tau_{0}^{\prime}=\omega^{*} \tau_{0}, \\
& \tau^{0^{\prime}}=\omega^{*} \tau^{0}, \tau^{1^{\prime}}=\omega^{*} \tau^{1}, T^{\prime}=\frac{\beta_{1} T}{\rho c_{1}^{2}}, \\
& C^{\prime}=\frac{\beta_{2} C}{\rho c_{1}^{2}}, \Omega^{\prime}=\frac{\Omega}{\omega^{*}}, M=\frac{\sigma_{0} B_{0}^{2}}{\rho \omega^{*}}, \\
& c_{1}^{2}=\frac{\lambda+2 \mu}{\rho}, \omega^{* 2}=\frac{\lambda}{\left(\mu t^{2}+\rho \alpha\right)},
\end{aligned}
$$

where $c_{1}$ is longitudinal wave velocity in the media and $M$ is the Hartmann number or magnetic parameter respectively.

The displacement components $u_{1}\left(x_{1}, x_{3}, t\right)$ and $u_{3}\left(x_{1}, x_{3}, t\right)$ are related to the scalar potentials $\Phi\left(x_{1}, x_{3}, t\right)$ and $\Psi\left(x_{1}, x_{3}, t\right)$ in a dimensionless form as

$$
u_{1}=\frac{\partial \Phi}{\partial x_{1}}-\frac{\partial \Psi}{\partial x_{3}}, u_{3}=\frac{\partial \Phi}{\partial x_{3}}+\frac{\partial \Psi}{\partial x_{1}} .
$$

We define the Laplace and Fourier transform as

$$
\begin{aligned}
& \overline{\mathrm{f}}\left(x_{1}, x_{3}, s\right)=\int_{0}^{\infty} f\left(x_{1}, x_{3}, t\right) e^{-s t} d t, \\
& \widehat{\mathrm{f}}\left(\xi, x_{3}, s\right)=\int_{-\infty}^{\infty} \overline{\mathrm{f}}\left(x_{1}, x_{3}, s\right) e^{i \xi x_{1}} d x_{1}
\end{aligned}
$$

Making use of (14) in (5)-(7) with the aid of (9)-(13), (15) and applying the Laplace and Fourier transforms defined by (16) on the resulting equation (after simplification), we obtain

$$
\begin{aligned}
& \left\{A D^{10}+B D^{8}+C D^{6}+E D^{4}+F D^{2}+G\right\} \\
\times & \{\widehat{\Phi}, \widehat{\Psi}, \hat{T}, \hat{C}\}=0
\end{aligned}
$$

where $A, B, C, E, F$ and $G$ are given in Appendix (I).

The solution of the equation (17) satisfying the radiation conditions that $\widehat{\Phi}, \widehat{\Psi}, \hat{T}$ and $\hat{C}$ tend to zero as $x_{3}$ tends to infinity can be written as

$$
\begin{aligned}
& (\widehat{\Phi}, \widehat{\Psi}, \hat{T}, \hat{C})\left(x_{3}, \xi, s\right)= \\
= & \sum_{i=1}^{5}\left(1, R_{i}, S_{i}, P_{i}\right) A_{i} e^{-m_{i} x_{3}},
\end{aligned}
$$

where

$$
R_{i}=\sum_{i=1}^{5} \frac{\delta_{7}}{\left[\left(2 a_{9} \xi^{2}+1\right) m_{i}^{2}-a_{1} m_{i}^{4}-\left(\delta_{5}+\delta_{6} \xi^{4}+\xi^{2}\right)\right]},
$$

$$
\begin{aligned}
& {\left[\delta_{8}\left(m_{i}^{2}-\xi^{2}\right)\left(\delta_{11}-\delta_{12}\left(m_{i}^{2}-\xi^{2}\right)^{2}\right)\right.} \\
& S_{i}=\sum_{i=1}^{5} \frac{\left.+\delta_{10}\left(m_{i}^{2}-\xi^{2}\right)^{2}\right]}{\left[\left(m_{i}^{2}-\xi^{2}-\delta_{9}\right)\left(\delta_{11}-\delta_{12}\left(m_{i}^{2}-\xi^{2}\right)\right)\right.}, \\
& \left.-\delta_{2} \delta_{10}\left(m_{i}^{2}-\xi^{2}\right)\right] \\
& P_{i}=\sum_{i=1}^{5} \frac{-\left(m_{i}^{2}-\xi^{2}\right)^{2}\left\{\delta_{2} \delta_{8}+\left(m_{i}^{2}-\xi^{2}-\delta_{9}\right)\right\}}{\left(m_{i}^{2}-\xi^{2}-\delta_{9}\right)\left(\delta_{11}-\delta_{12}\left(m_{i}^{2}-\xi^{2}\right)\right)} \text {, } \\
& -\delta_{2} \delta_{10}\left(m_{i}^{2}-\xi^{2}\right)
\end{aligned}
$$

$i=1,2,3,4,5$.

\section{BOUNDARY CONDITIONS}

(i) Mechanical boundary conditions, we suppose that the boundary plane $x_{3}=0$ is subjected to ramp-type normal loading, which depends on the coordinate $x_{1}$ and the time $t$ of the form

$$
t_{33}=-F G(t) F\left(x_{1}\right),
$$

where

$$
\begin{gathered}
G(t)=\left\{\begin{array}{lr}
0 & t \leq 0 \\
\frac{t}{t_{0}} & 0<t \leq t_{0} \\
1 & t>t_{0}
\end{array}\right. \\
F\left(x_{1}\right)=H\left(R-\left|x_{1}\right|\right),
\end{gathered}
$$

where $H$ is Heaviside unit step function, $R$ and $F$ are constant and $t_{0}$ is the ramp-type parameter.

Applying Laplace and Fourier transforms defined by (16) on (19) with the aid of (20) and (21), we obtain

$$
\hat{F}(\xi)=\frac{2}{\xi} \sin (\xi R),
$$

$$
\hat{t}_{33}=-\hat{F}\left(\frac{\left(1-e^{-s t_{0}}\right)}{t_{0} s^{2}}\right) \hat{F}(\xi)=-\hat{F}_{1}(\xi, s) .
$$

(ii) Vanishing of tangential stress

$$
t_{31}=0 .
$$

(iii) Vanishing of tangential couple stress

$$
m_{32}=0 .
$$

(iv) Isothermal boundary condition, i.e.

$$
T=0 \text { at } x_{3}=0 .
$$

(v) Mass concentration boundary condition, we consider the boundary plane $x_{3}=0$ is iso-concentrated surface, so

$$
C=0 .
$$


where

$$
\begin{gathered}
t_{33}=\frac{\lambda}{\beta_{1} T_{0}}\left[\left(\frac{\partial u_{1}}{\partial x_{1}}\right)+\left(1+\frac{2 \mu}{\lambda}\right)\left(\frac{\partial u_{3}}{\partial x_{3}}\right)\right. \\
\left.-\frac{\rho c_{1}^{2}}{\lambda}\left\{\left(1+\tau_{1} \frac{\partial}{\partial t}\right) T+\left(1+\tau^{1} \frac{\partial}{\partial t}\right) C\right\}\right], \\
t_{31}=\frac{\mu}{\beta_{1} T_{0}}\left(\frac{\partial u_{1}}{\partial x_{3}}+\frac{\partial u_{3}}{\partial x_{1}}\right)-\frac{\alpha \omega^{* 2}}{4 c_{1}^{2} \beta_{1} T_{0}} \\
\times\left[\left(\frac{\partial^{2}}{\partial x_{1}^{2}}+\frac{\partial^{2}}{\partial x_{3}^{2}}\right)\left(\frac{\partial u_{1}}{\partial x_{3}}-\frac{\partial u_{3}}{\partial x_{1}}\right)\right], \\
m_{32}=\frac{\alpha \omega^{* 2}}{2 c_{1}^{2} \beta_{1} T_{0}}\left(\frac{\partial^{2} u_{1}}{\partial x_{3}^{2}}-\frac{\partial^{2} u_{3}}{\partial x_{1} \partial x_{3}}\right) .
\end{gathered}
$$

Making use of (18) in the boundary conditions (19)-(27) and with the aid of (15), (16) and (28)-(30), we obtain the expressions for components of displacement, stress, temperature change and mass concentration as

$$
\begin{gathered}
\hat{u}_{1}=\frac{-\hat{F}_{1}(\xi, s)}{\triangle}\left[K_{1} \triangle_{1} e^{-m_{1} x_{3}}-K_{2} \triangle_{2} e^{-m_{2} x_{3}}\right. \\
\left.+K_{3} \triangle_{3} e^{-m_{3} x_{3}}-K_{4} \triangle_{4} e^{-m_{4} x_{3}}+K_{5} \triangle_{5} e^{-m_{5} x_{3}}\right] \\
\hat{u}_{3}=\frac{\hat{F}_{1}(\xi, s)}{\triangle}\left[T_{1} \triangle_{1} e^{-m_{1} x_{3}}-T_{2} \triangle_{2} e^{-m_{2} x_{3}}\right. \\
\left.+T_{3} \triangle_{3} e^{-m_{3} x_{3}}-T_{4} \triangle_{4} e^{-m_{4} x_{3}}+T_{5} \triangle_{5} e^{-m_{5} x_{3}}\right] \\
\hat{t}_{33}=-b_{1} \frac{\hat{F}_{1}(\xi, s)}{\triangle}\left[L_{1} \triangle_{1} e^{-m_{1} x_{3}}-L_{2} \triangle_{2} e^{-m_{2} x_{3}}\right. \\
\left.+L_{3} \triangle_{3} e^{-m_{3} x_{3}}-L_{4} \triangle_{4} e^{-m_{4} x_{3}}+L_{5} \triangle_{5} e^{-m_{5} x_{3}}\right] \\
\hat{t}_{31}=\frac{-\hat{F}_{1}(\xi, s)}{\triangle}\left[M_{1} \triangle_{1} e^{-m_{1} x_{3}}-M_{2} \triangle_{2} e^{-m_{2} x_{3}}\right. \\
\left.+M_{3} \triangle_{3} e^{-m_{3} x_{3}}-M_{4} \triangle_{4} e^{-m_{4} x_{3}}+M_{5} \triangle_{5} e^{-m_{5} x_{3}}\right] \\
\hat{m}_{32}=-V_{3} \frac{\hat{F}_{1}(\xi, s)}{\triangle}\left[N_{1} \triangle_{1} e^{-m_{1} x_{3}}-N_{2} \triangle_{2} e^{-m_{2} x_{3}}\right. \\
\left.+N_{3} \triangle_{3} e^{-m_{3} x_{3}}-N_{4} \triangle_{4} e^{-m_{4} x_{3}}+\triangle_{5} e^{-m_{5} x_{3}}\right]
\end{gathered}
$$

$$
\begin{gathered}
\hat{T}=-\frac{\hat{F}_{1}(\xi, s)}{\triangle}\left[S_{1} \triangle_{1} e^{-m_{1} x_{3}}-S_{2} \triangle_{2} e^{-m_{2} x_{3}}\right. \\
\left.+S_{3} \triangle_{3} e^{-m_{3} x_{3}}-S_{4} \triangle_{4} e^{-m_{4} x_{3}}+S_{5} \triangle_{5} e^{-m_{5} x_{3}}\right], \\
\hat{C}=-\frac{\hat{F}_{1}(\xi, s)}{\triangle}\left[P_{1} \triangle_{1} e^{-m_{1} x_{3}}-P_{2} \triangle_{2} e^{-m_{2} x_{3}}\right. \\
\left.+P_{3} \triangle_{3} e^{-m_{3} x_{3}}-P_{4} \triangle_{4} e^{-m_{4} x_{3}}+P_{5} \triangle_{5} e^{-m_{5} x_{3}}\right],
\end{gathered}
$$

where $\triangle, \triangle_{i}, K_{i}, T_{i}, L_{i}, M_{i}, N_{i}, b_{1}, V_{1}, V_{2}$ and $V_{3}$ are given in Appendix (II).

\section{PARTICULAR CASES}

(i) If $m=0$, in equations (31)-(37), we obtain the components of displacement and stresses in a modified couple stress thermoelastic with mass diffusion with rotating medium without Hall current effect.

(ii) If the effect of rotation is absent $(\Omega=0)$, in equations (31)-(37), we obtain the components of displacement and stresses in a modified couple stress thermoelastic with mass diffusion with the following changed values of $\delta_{1}, \delta_{4}, \delta_{5}$ and $\delta_{7}$ as

$$
\begin{aligned}
& \delta_{1}=s^{2}+\frac{M}{\left(1+m^{2}\right)} s-\Omega^{2}, \\
& \delta_{4}=\mathrm{S}\left[\frac{M m}{\left(1+m^{2}\right)}+2 \Omega\right], \\
& \delta_{5}=-\frac{1}{a_{1}}\left(\frac{M}{\left(1+m^{2}\right)} s+s^{2}-\Omega^{2}\right), \\
& \delta_{7}=-\frac{s}{a_{1}}\left[\frac{M m}{\left(1+m^{2}\right)}+2 \Omega\right] .
\end{aligned}
$$

(iv) If $\tau_{1}=\tau^{1}=0, \eta_{0}=1, \gamma=\tau_{0}$ in equations (31)-(37), we obtain the corresponding results for modified couple stress thermoelastic with mass diffusion under the influence of Hall current and rotation for the Lord Shulman (L-S) model.

(v) If $\eta_{0}=0, \gamma=\tau^{0}$ in equations (31)-(37), we obtain the corresponding results for the modified couple stress thermoelastic with mass diffusion under the influence of Hall current and rotation for the Green Lindsay (G-L) model.

\section{INVERSION OF THE TRANSFORMATION}

To obtain the solution of the problem in a physical domain, we must invert the transforms in (31)-(37). Here the displacement components, normal and tangential stresses and temperature change are functions of $x_{3}$, the parameters of the Laplace and Fourier transforms sand $\xi$, respectively and hence are of the form $f\left(\xi, x_{3}, s\right)$. To obtain the function $f\left(x_{1}, x_{3}, t\right)$ in the physical domain, we first invert the Fourier transform using 


$$
\begin{aligned}
& \overline{\mathrm{f}}\left(x_{1}, x_{3}, s\right)=\int_{-\infty}^{\infty} e^{-i \xi x_{1}} \widehat{\mathrm{f}}\left(\xi, x_{3}, s\right) d \xi= \\
= & \int_{-\infty}^{\infty}\left(\cos (\xi x) f_{e}-i \sin (\xi x) f_{o}\right) d \xi
\end{aligned}
$$

where $f_{e}$ and $f_{o}$ are, respectively, the odd and even points of $\hat{f}\left(\xi, x_{3}, s\right)$. Thus the expression (38) gives the Laplace transform $\overline{\mathrm{f}}\left(x_{1}, x_{3}, s\right)$ of the function $f\left(x_{1}, x_{3}, t\right)$. Following Honig and Hirdes [38], the Laplace transform function $\overline{\mathrm{f}}\left(x_{1}, x_{3}, s\right)$ can be inverted to $f\left(x_{1}, x_{3}, t\right)$.

The last step is to calculate the integral in equation (38). The method for evaluating this integral is described by Press et al. [39]. It involves the use of Romberg's integration with adaptive step size. This also uses the results from successive refinements of the extended trapezoidal rule followed by extrapolation of the results to the limit when the step size tends to zero.

\section{NUMERICAL RESULTS AND DISCUSSION}

For numerical computations, following Sherief and Saleh [30], we take the copper material (thermoelastic diffusion solid) as:

$$
\begin{aligned}
& \lambda=7.76 \times 10^{10} \mathrm{Kg} \mathrm{m}^{-1} \mathrm{~s}^{-2}, \\
& \mu=3.86 \times 10^{10} \mathrm{Kg} \mathrm{m}^{-1} \mathrm{~s}^{-2}, \\
& T_{0}=0.293 \times 10^{3} \mathrm{~K}, \\
& c_{e}=0.3831 \times 10^{3} \mathrm{~J} \mathrm{Kg}^{-1} \mathrm{~K}^{-1}, \\
& \alpha_{t}=1.78 \times 10^{-5} \mathrm{~K}^{-1},
\end{aligned}
$$

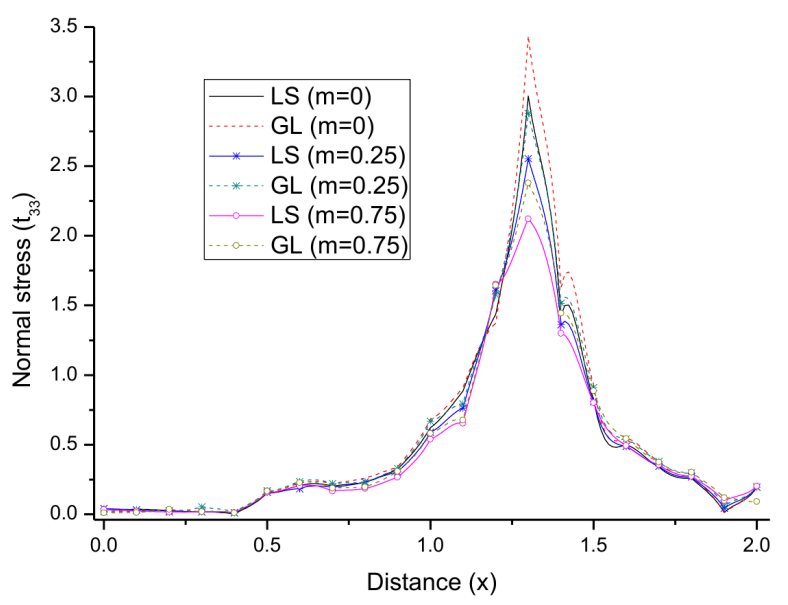

Fig. 1. Variation of normal stress with Hall parameter $m$

$$
\begin{aligned}
& \alpha_{c}=1.98 \times 10^{-4} \mathrm{~m}^{3} \mathrm{Kg}^{-1}, \\
& a=1.02 \times 10^{4} \mathrm{~m}^{2} \mathrm{~s}^{-2} \mathrm{~K}^{-1}, \\
& b=9 \times 10^{5} \mathrm{Kg}^{-1} \mathrm{~m}^{5} \mathrm{~s}^{-2}, \\
& D=0.85 \times 10^{-8} \mathrm{Kg} \mathrm{s} \mathrm{m}^{-3}, \\
& \rho=8.954 \times 10^{3} \mathrm{Kg} \mathrm{m}^{-3}, \\
& K^{*}=0.383 \times 10^{3} \mathrm{~W} \mathrm{~m}^{-1} \mathrm{~K}^{-1}, \\
& \alpha=.05 \mathrm{Kg} \mathrm{m} \mathrm{s}^{-2}, \\
& t=0.5 \mathrm{~s}, \\
& \tau_{0}=0.01 \mathrm{~s}, \\
& \tau^{0}=0.02 \mathrm{~s}, \\
& \tau_{1}=0.07 \mathrm{~s}, \\
& \tau^{1}=0.08 \mathrm{~s} .
\end{aligned}
$$

The Hall current parameters are taken from Zakaria [37]

$$
\begin{gathered}
\sigma_{0}=9.36 \times 10^{5} \mathrm{Col}^{2} \mathrm{sec} / \mathrm{Kg} \mathrm{m}^{3}, \\
H_{0}=10^{5} \mathrm{Col} / \mathrm{m} \mathrm{sec}, B_{0}=0.5 \mathrm{KgCol}^{-1} \mathrm{sec}^{-1}
\end{gathered}
$$

The software Matlab 7.10.4 has been used to determine the normal stress, tangential stress, couple stress, temperature change and mass concentration for different values of Hall current parameters and rotation for both L-S and G-L theories are computed numerically and shown graphically in Figs. $1-10$, respectively.

In Figs. 1-5, solid line (-), solid line with centre symbol $(-*-)$ and solid line with centre symbol $(-O-)$ correspond to the L-S theory for $m=0,0.5,1.5$ and keeping $\Omega=0.5$, respectively. Similarly, small dash line (- - - ), small dash line with the centre symbol (- - - - - - ) and small dash line with the centre symbol (- - -o- - -) correspond to the G-L theory for $m=0,0.25,0.75$ and keeping $\Omega=0.5$, respectively.

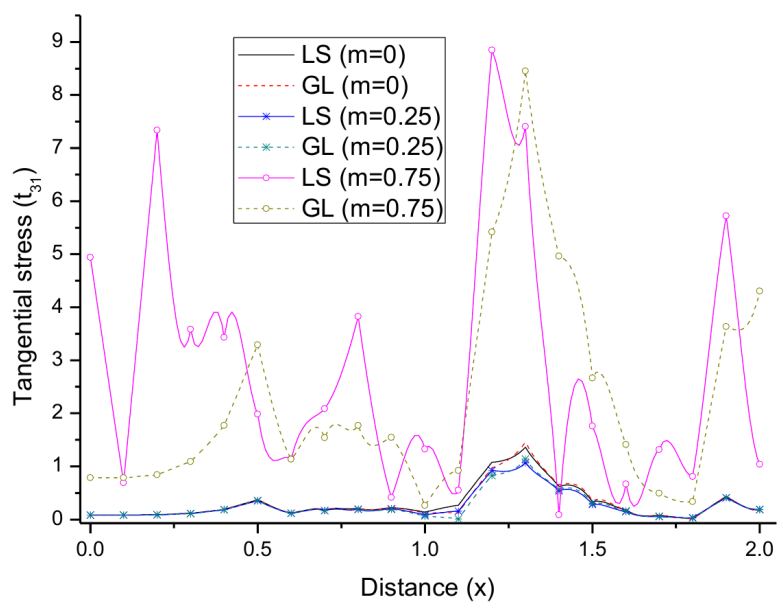

Fig. 2. Variation of tangential stress with Hall parameter $m$ 


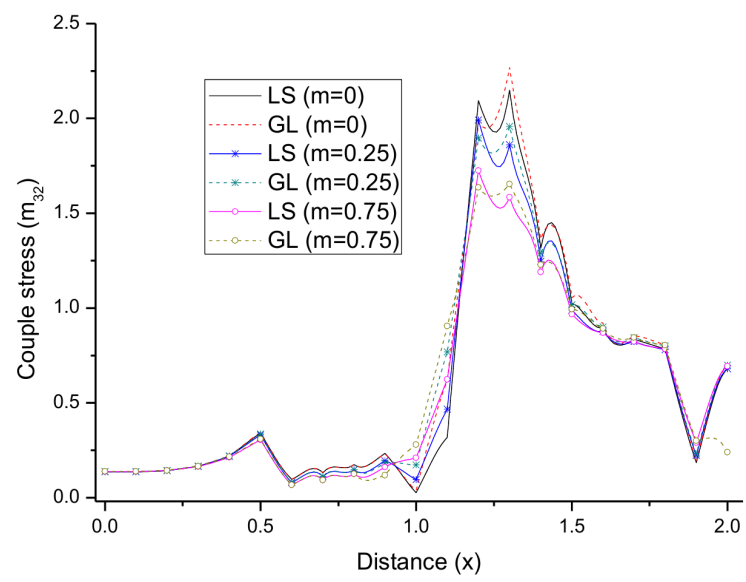

Fig. 3. Variation of couple stress with Hall parameter $m$

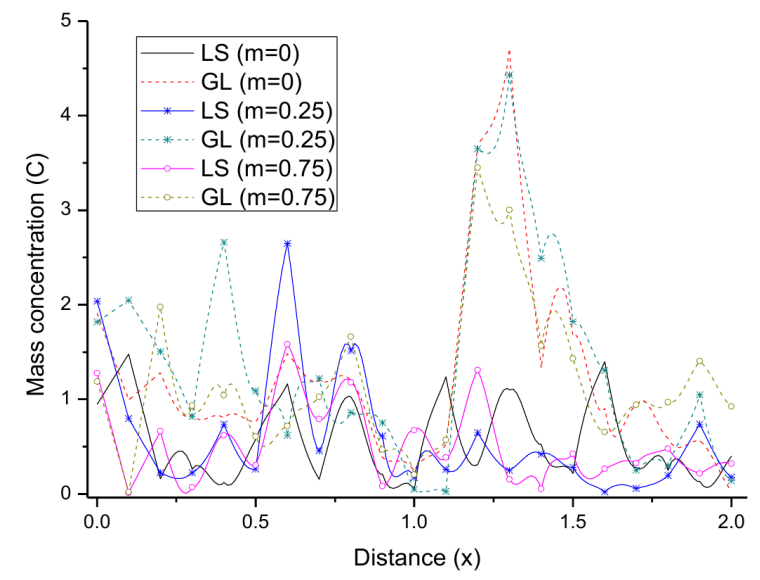

Fig. 5. Variation of mass concentration with Hall parameter $m$

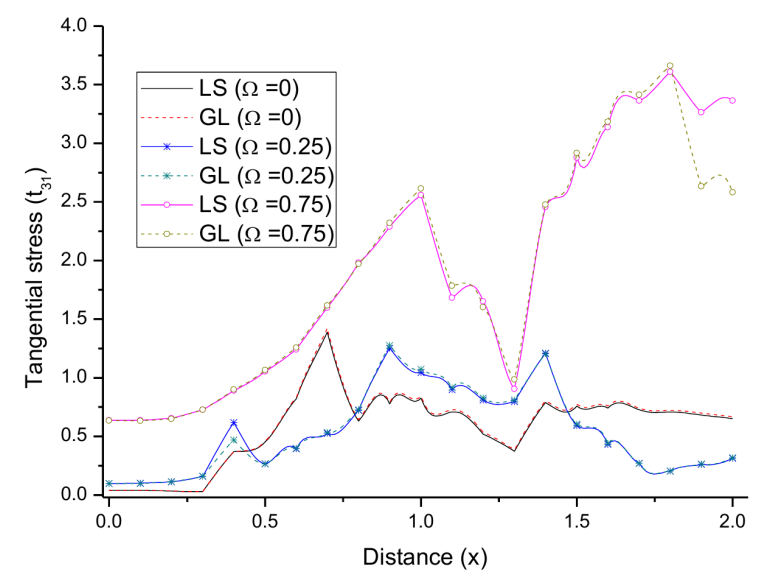

Fig. 7. Variation of tangential stress with rotation $\Omega$

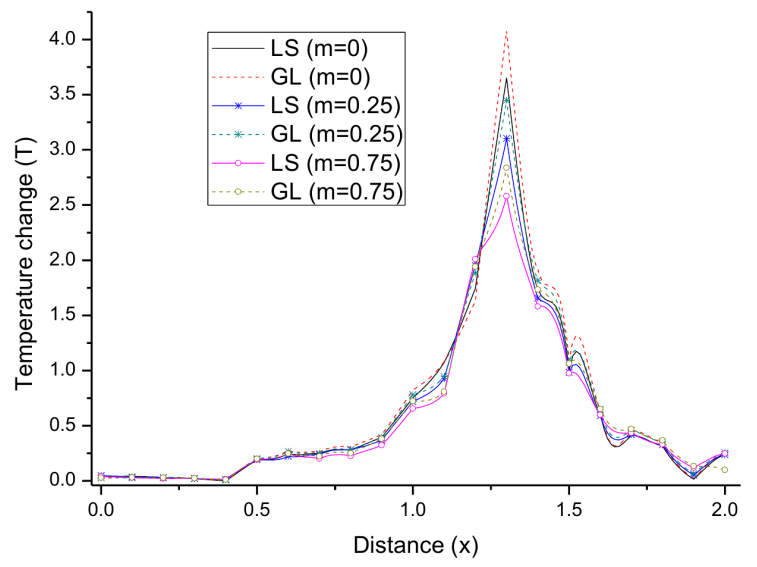

Fig. 4. Variation of temperature change with Hall parameter $m$

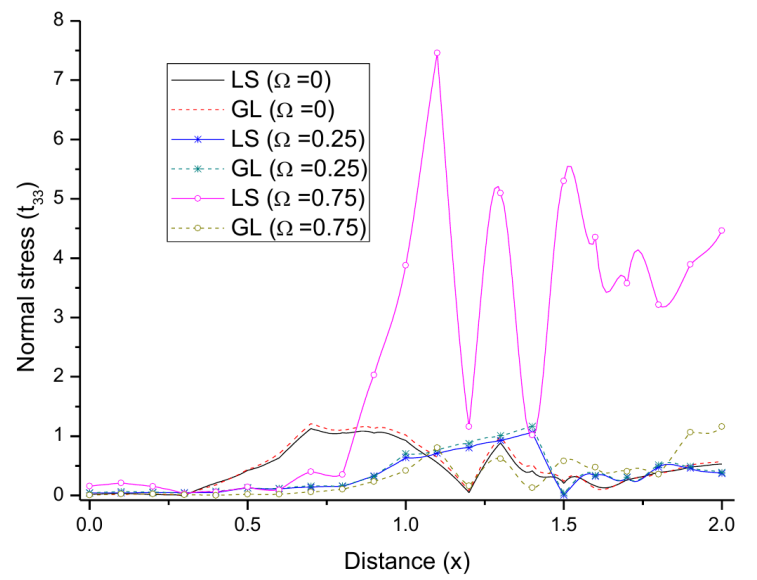

Fig. 6. Variation of normal stress with rotation $\Omega$

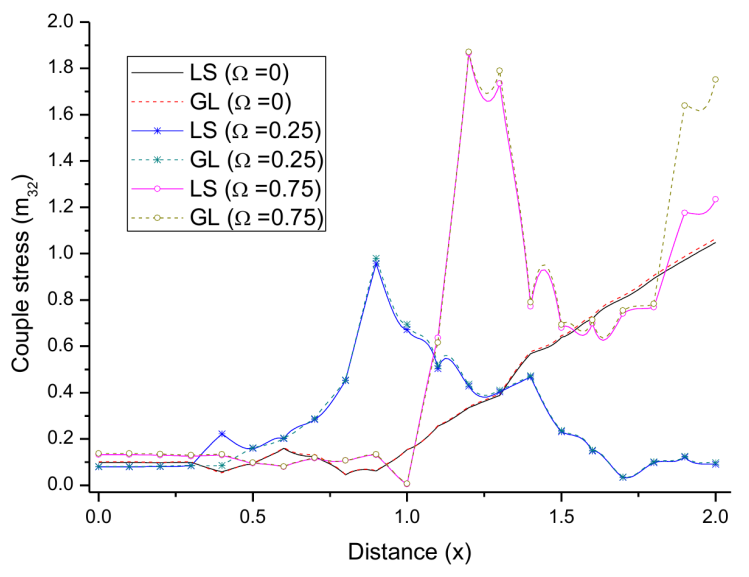

Fig. 8. Variation of couple stress with rotation $\Omega$ 


\section{Effect of Hall current}

From Figs. 6-10, solid line (-), solid line with the centre symbol (-*-) and solid line the with centre symbol $(-O-)$ correspond to the L-S theory for $\Omega=0,0.25,0.75$ and keeping $\mathrm{m}=0.5$, respectively. Similarly, small dash line (- - -), small dash line with the centre symbol (- - -*- - -) and small dash line with the centre symbol (- - - - - -) correspond to the G-L theory for $\Omega=0,0.25,0.75$ and keeping $m=0.5$, respectively.

Fig. 1 shows the variations of normal stress $t_{33}$ with distance $x$ for both L-S and G-L theories for different values of Hall parameter. Its values initially oscillate in the range $0 \leq x<0.7$, increase monotonically in the range $0.7 \leq x<1.4$ and then decrease rapidly as $x$ increase further. The values of normal stress for the G-L theory is higher in comparison to the L-S theory for all values of $m=0,0.25,0.75$. The value of normal stress increases in the absence of Hall current and decreases in the presence of Hall current for both theories of thermoelasticity.

Fig. 2 represents the variations of tangential stress with different values of Hall parameter $m=0,0.25,0.75$. It is noticed that the values of $t_{31}$ first oscillate in the range $0 \leq x<1.1$, increase rapidly in the range $1.1 \leq x<1.4$, decrease in the range $1.4 \leq x \leq 1.8$ and then again oscillate for the remaining values of $x$ for $m=0,0.25$ and for both the theories of thermoelasticity. Also for $m=0.75$, the values of tangential stress for L-S theory are higher in comparison to the G-L theory. As Hall current parameter increases, tangential stress also increases in the assumed region for both L-S and G-L theories.

Fig. 3 depicts that the variations of couple stress $m_{32}$ with distance $x$ for Hall parameter $m=0,0.25,0.75$.Its values initially oscillate in the range $0 \leq x \leq 1.0$, increase sharply in the range $1.0 \leq x \leq 1.3$, decrease rapidly in the range $1.3 \leq x \leq 1.8$ and then increase further for remaining values of $x$. The values of $m_{32}$ remain oscillatory for all values of $x$ for both L-S and G-L theories. The values of couple stress remain oscillatory in the entire region under the absence and presence of Hall current.

Fig. 4 shows the variations of Temperature change $T$ with distance $x$ for both L-S and G-L theories for different values of Hall parameter. The values of $T$ increase monotonically in the range $0 \leq x \leq 1.3$ then decrease as $x$ increase further. Also the values of temperature change for the G-L theory are higher in comparison to the L-S theory as the Hall parameter increases. The values of temperature change is higher for $m=0$ and smaller for $m=0.25,0.75$.

Fig. 5 represents the variations of mass concentration $C$ with Hall parameter. The values of concentration for the G-L theory are higher in the range $0 \leq x<0.6$, lower in the range $0.6 \leq x<1.2$ and again higher for remaining values of $x$ in comparison to the L-S theory for $m=0,0.25,0.75$. The behavior and variation of mass concentration is oscillatory in the absence and presence of Hall current.

\section{Effect of rotation}

Fig. 6 depicts the variations of normal stress with distance $x$ for rotation $(\Omega=0,0.25,0.75)$. For $\Omega=0,0.25$, the values of $t_{33}$ for G-L theory are higher than in comparison to the L-S theory in the whole range, whereas reverse behavior is observed for $\Omega=0.75$. Oscillatory behavior is observed with and without rotation.

Fig. 7 shows that the variations of tangential stress with distance $x$ for rotation $\Omega=0,0.25,0.75$. The values of tangential stress for $\Omega=0.75$ are higher than that of $\Omega=0,0.25$. Also, the values of $t_{31}$ for G-L theory are higher in comparison to the L-S theory in the range $0 \leq x<2.1$ for all values of rotation. The value of tangential stress increases with increase in the values of rotation.

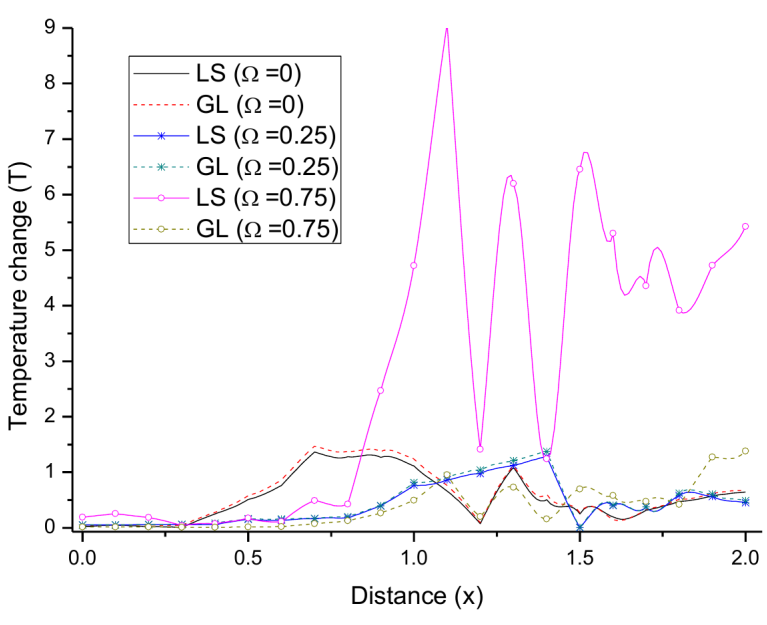

Fig. 9. Variation of temperature Changewith rotation $\Omega$

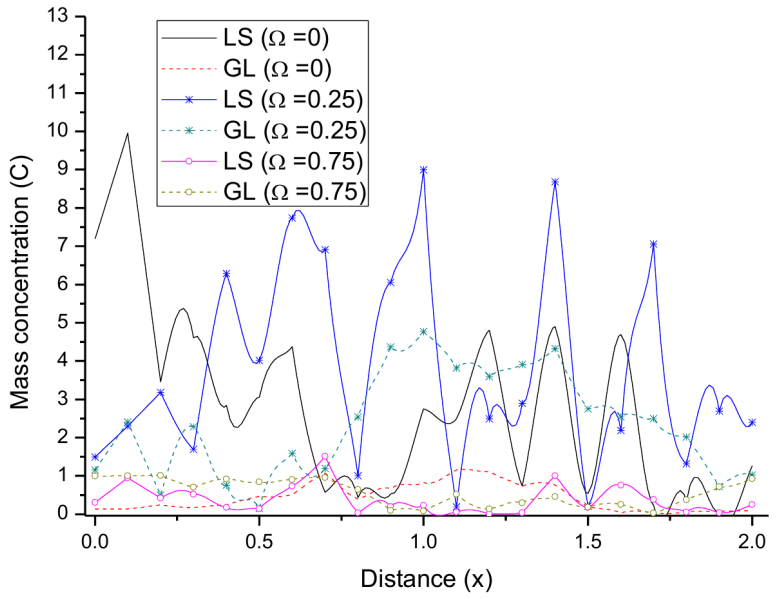

Fig. 10. Variation of mass concentration with rotation $\Omega$ 
Fig. 8 represents the variations of couple stress $m_{32}$ with different values of rotation $\Omega=0,0.25,0.75$. For $\Omega=0$, the values of couple stress initially oscillate in the range $0 \leq x<1$ and then increase for remaining values of $x$. Similarly, the values of $m_{32}$ for $\Omega=0.25$, increase monotonically in the range $0 \leq x<1$, decrease in the range $1 \leq x<1.4$ and then oscillate as $x$ increase further and then opposite behavior is noticed for $\Omega=0.75$. The values of couple stress for G-L theories are greater than in comparison to L-S theories for $\Omega=0,0.25$ and 0.75 . The value of Couple stress decreases in the absence of rotation and increases in the presence of rotation.

Fig. 9 represents the variations of $T$ with different values of rotation for both theories of thermoelasticity. Its values first oscillate in the range $0 \leq x<1.1$, increase monotonically in the range $1.1 \leq x<1.4$ and decrease rapidly in the remaining values of $x$. The values of temperature change for the G-L theory are higher in comparison to the L-S theory for $\Omega=0,0.25,0.75$. The value of temperature change increase and decrease alternatively with and without rotation.

Fig. 10 depicts the variations of mass concentration $C$ with distance $x$ for both L-S and G-L theories for $\Omega=$ $0,0.25,0.75$. The behavior of variations of concentration for L-S theory is higher in comparison to the G-L theory for $\Omega=0$, whereas for $\Omega=0.25$, the values of $C$ for L-S are higher in the range $0 \leq x<1.1$, lower in the range $1.1 \leq x \leq 1.8$ and then again higher in the remaining values of $x$. Similarly, the values of concentration for $\Omega=0.75$, increase and decrease alternately in the whole range for both $\mathrm{L}-\mathrm{S}$ and G-L theories. The behavior of mass concentration is oscillatory in the absence and presence of rotation for both theories of thermoelasticity.

\section{CONCLUSIONS}

Analysis of stresses, temperature change and mass concentration due to ramp-type loading is a significant problem of continuum mechanics. The result obtained from the above study are summarized as.

The resulting quantities depicted graphically are observed to be very sensitive towards the Hall and rotation parameters. Figures show that the Hall and rotation parameters have oscillatory effects on the numerical values of the physical quantities obtained after the computational process. It is also observed that the physical quantities are also effected by the different non-classical theories of thermoelasticity.

It is observed that initially the values of $t_{33}$ and $T$ for G-L theory are higher in comparison to L-S theory as the Hall parameter increases and reverse behavior is noticed due to the effect of rotation, whereas the values of $t_{31}$ for L-S theory are higher in comparison to G-L theory as the Hall parameter increases and decreases under the effect of rotation.

It is also noticed that the values of $m_{32}$ for the G-L theory are higher in comparison to the L-S theory due to the effect of Hall parameter and rotation. Appreciable effects of Hall parameter and rotation are observed on the mass concentration.

The results obtained in the study should be beneficial for people working on modified couple stress thermoelastic solid with mass diffusion. By introducing the Hall parameter and rotation to the assumed model present a more realistic mode for future study.

\section{APPENDIX I}

$$
\begin{aligned}
& A=a_{1}\left(\delta_{12}-\delta_{3}\right), \\
& B=-a_{1} \delta_{12}\left(\xi^{2}+\delta_{1}\right)-\left(\left(\delta_{12}-\delta_{3}\right)\left(2 a_{9} \xi^{2}+1\right)\right. \\
& +a_{1}\left(\delta_{11}+\delta_{12}\left(2 \xi^{2}+\delta_{9}\right)-\delta_{2} \delta_{10}-2 \xi^{2} \delta_{3}\right) \\
& \left.-\left(\delta_{10}-\delta_{8} \delta_{12}\right)+\delta_{3}\left(\delta_{2} \delta_{8}+\xi^{2}+\delta_{9}\right)\right) \text {, } \\
& C=\left(\xi^{2}+\delta_{1}\right)\left(\delta_{12}\left(2 a_{9} \xi^{2}+1\right)+a_{1}\left(\delta_{11}+\delta_{12}\left(2 \xi^{2}+\delta_{9}\right)\right.\right. \\
& \left.\left.-\delta_{10} \delta_{2}\right)\right)+\left(2 a_{9} \xi^{2}+1\right)\left(\delta_{11}-\delta_{2} \delta_{10}+\delta_{12}\left(2 \xi^{2}+\delta_{9}\right)\right) \\
& +a_{1}\left(\xi^{2}\left(\delta_{11}+\delta_{9} \delta_{12}+\xi^{2} \delta_{12}\right)+\delta_{9} \delta_{11}\right)+\delta_{12}\left(\delta_{5}+\xi^{2}\right. \\
& \left.+\delta_{6} \xi^{4}\right)+a_{1}\left(\delta_{8} \delta_{11}-\delta_{2} \delta_{10} \xi^{2}\right)+\left(\delta_{8} \delta_{12}-\delta_{10}\right)\left(\left(2 a_{9} \xi^{2}\right.\right. \\
& \left.+1)+2 a_{1} \xi^{2}\right)+\delta_{3}\left(\left(\delta_{2} \delta_{8}-\xi^{2}-\delta_{9}\right)\left(2 a_{9} \xi^{2}+1\right)\right. \\
& \left.-\left(\delta_{5}+\xi^{2}+\delta_{6} \xi^{4}\right)-a_{1} \xi^{4}\right)-2 \xi^{2} \delta_{3}\left(\left(2 a_{9} \xi^{2}+1\right)\right. \\
& \left.-a_{1}\left(\delta_{8} \delta_{2}+\xi^{2}+\delta_{9}\right)\right) \text {, } \\
& E=-\left(\xi^{2}+\delta_{1}\right)\left\{( \delta _ { 1 1 } + \delta _ { 1 2 } ( 2 \xi ^ { 2 } + \delta _ { 9 } ) - \delta _ { 1 0 } \delta _ { 2 } ) \left(2 a_{9} \xi^{2}\right.\right. \\
& +1)+\delta_{12}\left(\delta_{5}+\xi^{2}+\delta_{6} \xi^{4}\right)+a_{1}\left(\xi ^ { 2 } \left(\delta_{11}-\delta_{10} \delta_{2}\right.\right. \\
& \left.\left.\left.+\delta_{12}\left(\delta_{9}+\xi^{2}\right)\right)+\delta_{9} \delta_{11}\right)\right\}+\left(2 a_{9} \xi^{2}+1\right)\left(\delta_{12} \xi^{4}\right. \\
& \left.+\left(\delta_{2}\left(1-\delta_{10}\right)+\delta_{9} \delta_{12}\right) \xi^{2}+\delta_{9} \delta_{11}\right)-\left(\delta_{5}+\xi^{2}+\delta_{6} \xi^{4}\right) \\
& \times\left(\delta_{11}+\delta_{12}\left(2 \xi^{2}+\delta_{9}\right)-\delta_{10} \delta_{2}\right)+\delta_{4} \delta_{7} \delta_{12} \\
& +\left(2 a_{9} \xi^{2}+1\right)\left(2 \xi^{2}\left(\delta_{10}-\delta_{8} \delta_{12}\right)-\delta_{8} \delta_{11}\right) \\
& +\left(\delta_{5}+\xi^{2}+\delta_{6} \xi^{4}\right)\left(\left(\delta_{10}-\delta_{8} \delta_{12}\right)\right. \\
& \left.-\left(\delta_{2} \delta_{8}-\xi^{2}-\delta_{9}\right)+2 \xi^{2}\right)+a_{1} \xi^{2}\left(\xi ^ { 2 } \left(\delta_{10}\right.\right. \\
& \left.\left.-\delta_{8} \delta_{12}\right)-\delta_{8} \delta_{11}\right)+\xi^{4}\left(\left(2 a_{9} \xi^{2}+1\right)\right. \\
& \left.-a_{1}\left(\delta_{2} \delta_{8}+\xi^{2}+\delta_{9}\right)\right)-2 \xi^{2}\left(2 a_{9} \xi^{2}+1\right) \\
& \times\left(\delta_{2} \delta_{8}-\xi^{2}-\delta_{9}\right) \text {, } \\
& F=-\left(\xi^{2}+\delta_{1}\right)\left\{\left(-\delta_{12} \xi^{4}+\left(\delta_{2} \delta_{10}\right.\right.\right. \\
& \left.\left.-\delta_{9} \delta_{12}-\delta_{11}\right) \xi^{2}-\delta_{9} \delta_{11}\right)\left(2 a_{9} \xi^{2}+1\right) \\
& \left.-\left(\delta_{5}+\xi^{2}+\delta_{6} \xi^{4}\right)\left(\delta_{11}+\delta_{12}\left(2 \xi^{2}+\delta_{9}\right)-\delta_{2} \delta_{10}\right)\right\} \\
& +\left(\delta_{5}+\xi^{2}+\delta_{6} \xi^{4}\right)\left(\delta_{12} \xi^{4}+\left(\delta_{11}+\delta_{9} \delta_{12}-\delta_{2} \delta_{10}\right) \xi^{2}\right. \\
& \left.+\delta_{9} \delta_{11}\right)-\delta_{4} \delta_{7}\left(\delta_{11}+\delta_{12}\left(2 \xi^{2}+\delta_{9}\right)-\delta_{2} \delta_{10}\right)+
\end{aligned}
$$




$$
\begin{aligned}
& +\left(2 a_{9} \xi^{2}+1\right)\left(\delta_{8} \delta_{11} \xi^{2}+\left(\delta_{8} \delta_{12}-\delta_{10}\right) \xi^{4}\right) \\
& +\left(\delta_{5}+\xi^{2}+\delta_{6} \xi^{4}\right)\left(\delta_{8} \delta_{11}+2 \xi^{2}\left(\delta_{8} \delta_{12}-\delta_{10}\right)\right) \\
& +\xi^{4}\left(\left(\delta_{2} \delta_{8}-\xi^{2}-\delta_{9}\right)\left(2 a_{9} \xi^{2}+1\right)-\left(\delta_{5}+\xi^{2}+\delta_{6} \xi^{4}\right)\right) \\
& +2 \xi^{2}\left(\delta_{2} \delta_{8}-\xi^{2}-\delta_{9}\right)\left(\delta_{5}+\xi^{2}+\delta_{6} \xi^{4}\right), \\
& \quad \\
& \quad=\left(\delta_{12} \xi^{4}+\left(\delta_{11}+\delta_{9} \delta_{12}-\delta_{2} \delta_{10}\right) \xi^{2}+\delta_{9} \delta_{11}\right) \\
& \quad \times\left(\delta_{4} \delta_{7}-\left(\xi^{2}+\delta_{1}\right)\left(\delta_{5}+\xi^{2}+\delta_{6} \xi^{4}\right)\right) \\
& \quad+\left(\delta_{5}+\xi^{2}+\delta_{6} \xi^{4}\right)\left(-\delta_{8} \delta_{11} \xi^{2}+\xi^{4}\left(\left(\delta_{10}-\delta_{8} \delta_{12}\right)\right.\right. \\
& \left.\left.\quad-\left(\delta_{2} \delta_{8}-\xi^{2}-\delta_{9}\right)\right)\right),
\end{aligned}
$$

and

$$
\begin{aligned}
& a_{1}=\frac{\mu}{\rho c_{1}^{2}}, a_{2}=\frac{\alpha \omega^{* 2}}{4 \rho c_{1}^{4}}, a_{3}=\frac{\rho c_{e} c_{1}^{2}}{K^{*} \omega^{*}}, a_{4}=\frac{a T_{0} \beta_{1} c_{1}^{2}}{\beta_{2} K^{*} \omega^{*}}, \\
& a_{5}=\frac{T_{0} \beta_{1}^{2}}{\rho K^{*} \omega^{*}}, a_{6}=\frac{a \rho c_{1}^{2}}{\beta_{1} \beta_{2}}, a_{7}=\frac{\rho c_{1}^{4}}{\beta_{2}^{2} D \omega^{*}}, a_{8}=\frac{b \rho c_{1}^{2}}{\beta_{2}^{2}} \\
& \tau_{t}=\left(1+\tau_{1} \frac{\partial}{\partial t}\right), \tau_{t}^{1}=\left(1+\tau^{1} \frac{\partial}{\partial t}\right), \\
& \tau_{\eta_{0}}^{0}=\left(\frac{\partial}{\partial t}+\eta_{0} \tau_{0} \frac{\partial^{2}}{\partial t^{2}}\right), \tau_{t}^{0}=\left(\frac{\partial}{\partial t}+\tau_{0} \frac{\partial^{2}}{\partial t^{2}}\right), \\
& \tau_{\gamma}^{0}=\left(\frac{\partial}{\partial t}+\gamma \frac{\partial^{2}}{\partial t^{2}}\right), \tau_{t}^{10}=\left(\frac{\partial}{\partial t}+\eta_{0} \tau^{0} \frac{\partial^{2}}{\partial t^{2}}\right), \\
& e=\nabla^{2} \Phi, \frac{\partial u_{1}}{\partial x_{3}}-\frac{\partial u_{3}}{\partial x_{1}}=-\nabla^{2} \Psi . \\
& a_{9}=\frac{a_{2}}{a_{1}}, \delta_{1}=s^{2}+\frac{M}{\left(1+m^{2}\right)} s-\Omega^{2}, \delta_{2}=\left(1+\tau_{1} s\right), \\
& \delta_{3}=\left(1+\tau^{1} s\right), \delta_{4}=\mathrm{s}\left[\frac{M m}{\left(1+m^{2}\right)}+2 \Omega\right] \\
& \delta_{5}=\frac{1}{a_{1}}\left(\frac{M}{\left(1+m^{2}\right)} s+s^{2}-\Omega^{2}\right), \delta_{6}=\frac{1}{a_{9}}, \\
& \left.\left.\delta_{1}=m^{2}\right)+2 \Omega\right], \delta_{8}=a_{5}\left(s+\tau_{0} \eta_{0} s^{2}\right),
\end{aligned}
$$$$
\begin{aligned}
& \delta_{9}=a_{3}\left(s+\tau_{0} s^{2}\right), \delta_{10}=a_{4}\left(s+\gamma s^{2}\right), \\
& \delta_{11}=a_{7}\left(s+\tau^{0} \eta_{0} s^{2}\right), \delta_{12}=a_{8} \delta_{3} .
\end{aligned}
$$

\section{APPENDIX II}

$$
\begin{aligned}
\triangle & =g_{1}\left(L_{1} h_{2}-L_{2} h_{1}+L_{3} h_{3}\right) \\
& +g_{2}\left(L_{1} h_{4}-L_{2} h_{5}-L_{4} h_{3}\right) \\
& +g_{3}\left(L_{1} h_{6}-L_{2} h_{12}+L_{5} h_{3}\right) \\
& +g_{4}\left(L_{1} h_{8}+L_{3} h_{9}-L_{4} h_{10}\right) \\
& +g_{5}\left(L_{1} h_{11}+L_{3} h_{12}+L_{5} h_{10}\right) \\
& +g_{6}\left(L_{1} h_{14}-L_{5} h_{9}-L_{4} h_{12}\right) \\
& +g_{7}\left(L_{2} h_{14}-L_{4} h_{6}-L_{5} h_{4}\right) \\
& +g_{8}\left(L_{2} h_{8}+L_{3} h_{4}+L_{4} h_{2}\right) \\
& +g_{9}\left(L_{2} h_{11}+L_{3} h_{6}-L_{5} h_{2}\right) \\
& +g_{10}\left(L_{3} h_{14}+L_{4} h_{11}+L_{5} h_{8}\right),
\end{aligned}
$$

$\triangle_{i}(i=1, \ldots, 5)$ are obtain by replacing $1^{s t}, 2^{\text {nd }}, 3^{r d}$, $4^{\text {th }}$ and $5^{\text {th }}$ column by $\left[0,0,0, F_{1}(\xi, s), 0\right]^{T}$ in $\triangle_{i}$. and $K_{i}=\left(-i \xi+m_{i} R_{i}\right), T_{i}=\left(m_{i}+i \xi R_{i}\right), L_{i}=$ $\left(\left(m_{i}^{2}-\xi^{2}\right)+\frac{2 \mu}{\lambda} m_{i}\left(m_{i}+i \xi R_{i}\right)-\frac{\rho c_{1}^{2}}{\lambda}\left(\delta_{2} S_{i}+\delta_{3} P_{i}\right)\right)$,

$$
\begin{aligned}
M_{i} & =V_{1}\left(2 i \xi m_{i}-\left(m_{i}^{2}+\xi^{2}\right) R_{i}\right) \\
& -V_{2}\left(\left(m_{i}^{2}-\xi^{2}\right)\left(\left(m_{i} i \xi-R_{i} m_{i}^{2}\right)+i \xi\left(m_{i}+i \xi R_{i}\right)\right)\right), \\
N_{i} & =m_{i}\left(m_{i}^{2}-\xi^{2}\right) R_{i}, \\
b_{1} & =\frac{\lambda}{\beta_{1} T_{0}}, \\
V_{1} & =\frac{\mu}{\beta_{1} T_{0}}, \\
V_{2} & =\frac{\alpha \omega^{* 2}}{4 c_{1}^{2} T_{1} T_{0}} V_{3}=\frac{\alpha \omega^{* 2}}{2 c_{1}^{2} \beta_{1} T_{0}}, \\
g_{1} & =\left(S_{4} P_{5}-P_{4} S_{5}\right), \\
g_{2} & =\left(S_{3} P_{5}-P_{3} S_{5}\right), \\
g_{3} & =\left(S_{3} P_{4}-P_{3} S_{4}\right), \\
g_{4} & =\left(S_{3} P_{4}-P_{3} S_{4}\right), \\
g_{5} & =\left(S_{2} P_{4}-P_{2} S_{4}\right), \\
g_{6} & =\left(S_{2} P_{3}-P_{2} S_{3}\right), \\
g_{7} & =\left(S_{3} P_{1}-P_{3} S_{1}\right), \\
g_{8} & =\left(S_{5} P_{1}-P_{5} S_{1}\right), \\
g_{9} & =\left(S_{4} P_{1}-P_{4} S_{1}\right), \\
g_{10} & =\left(S_{1} P_{2}-P_{1} S_{2}\right) \\
h_{1} & =\left(M_{1} N_{3}-M_{3} N_{1}\right), h_{2}=\left(M_{2} N_{3}-M_{3} N_{2}\right), \\
h_{3} & =\left(M_{1} N_{2}-M_{2} N_{1}\right), h_{4}=\left(M_{4} N_{2}-M_{2} N_{4}\right), \\
h_{5} & =\left(M_{4} N_{1}-M_{1} N_{4}\right), h_{6}=\left(M_{2} N_{5}-M_{5} N_{2}\right), \\
h_{7} & =\left(M_{1} N_{5}-M_{5} N_{1}\right), h_{8}=\left(M_{3} N_{4}-M_{4} N_{3}\right), \\
h_{9} & =\left(M_{4} N_{1}-M_{1} N_{4}\right), h_{10}=\left(M_{3} N_{1}-M_{1} N_{3}\right), \\
h_{11} & =\left(M_{5} N_{3}-M_{3} N_{5}\right), h_{12}=\left(M_{4} N_{5}-M_{5} N_{4}\right) . \\
i & =1,2,3,4 .
\end{aligned}
$$

\section{References}

[1] R.A. Toupin, Elastic materials with couple-stresses. Arch. Rational Mech. and Anal., 11, 385-414, (1962). 
[2] R.D. Mindlin, H. F. Tiersten, Effects of couple-stresses in linear elasticity. Arch. Rational Mech. and Anal., 11, 415-448, (1962).

[3] W.T. Koiter, Couple-stresses in the theory of elasticity. Proc. R. Neth. Acad. Sci., 67, 17-44, (1964).

[4] J. Zhao, C. Wanji, B. Ji, A weak continuity condition of FEM for axisymmetric couple stress theory and an 18-DOF triangular axisymmetric element. Finite Elements in Analysis and Design, 46(8), 632-644, (2010).

[5] J.N. Reddy, Microstructure-dependent couple stress theories of functionally graded beams. J. Mech. Phys. Solids, 59, 23822399, (2011).

[6] A.R. Hadjesfandiari, G. F. Dargush, Boundary element formulation for plane problems in couple stress elasticity. Numerical methods in Engineering, 89(5), 618-636, (2012).

[7] F. Yang, A. C. M. Chong, D. C. C. Lam, P. Tong, Couple stress based strain gradient theory for elasticity. International Journal of Solids and Structures, 39, 2731-2743, (2002).

[8] M. R. Shankar, S. Chandrasekar, T. N., Farris Interaction between dis-locations in a couple stress medium. ASME J. Appl. Mech., 71, 546-550, (2004).

[9] C. Babaoglu, S. Erbay, Two-dimensional wave packets in an elastic solid with couple stresses. Int. J. Non-Linear Mech., 39, 941-949, (2004).

[10] S. Diebels, H. Steeb, Stress and couple stress in foams. Comput. Mater. Sci., 28, 714-722, (2003).

[11] M.A. Kulesh, V. P. Matveenko, I. N. Shardakov, Parametric analysis of analytical solutions to one and two dimensional problems in couple-stress theory of elasticity. ZAMM J. Appl. Math. Mech., 83, 238-248, (2003).

[12] S.K. Park, X. L. Gao, Bernoulli-Euler beam model based on a modified couple stress theory. J. of Micromech. and Micro engg., 16, 2355, (2006).

[13] H.M. Ma, X. L. Gao, J. N. Reddy, A microstructure-dependent Timoshenko beam model based on a modified couple stress theory. J. of the Mech. and Phys. of Solids, 56, 3379- 3391, (2008).

[14] M. Marin, On the minimum principle for dipolar materials with stretch. Nonlinear Analysis: Real World Applications, 10, 1572-1578, (2009).

[15] G.C. Tsiatas, A new Kirchhoff plate model based on a modified couple stress theory. International Journal of Solids and Structures, 46, 2757-2764, (2009).

[16] H.M. Ma, X. L. Gao, J. N. Reddy, A non-classical Mindlin plate model based on a modified couple stress theory. Acta. Mech. 220, 217-35, (2011).

[17] M. Asghari, Geometrically nonlinear micro-plate formulation based on the modified couple stress theory. Int. J. Engg. Sci., 51, 292-309, (2012).

[18] M. Marin and G. Stan, Weak solutions in elasticity of dipolar bodies with stretch. CARPATHIAN J. of Mathematics, 29(1), 33-40, (2013).

[19] M. Simsek, J. N. Reddy, Bending and vibration of functionally graded microbeams using a new higher order beam theory and the modified couple stress theory. Int. J. of Engg. Sci., 64, 37-53, (2013).

[20] M. Marin, R. P. Agarwal and S. R. Mahmoud, Nonsimple material probems addressed by the Lagrange's identity. Boundary Value Problems, Article No. 135, (2013), DOI: 10.1186/16872770-2013-135.
[21] M. Mohammad-Abadi, A. R. Daneshmehr, Size dependent buckling analysis of micro beams based on modified couple stress theory with high order theories and general boundary conditions. Int.J. of Engg. Sci., 74, 1-14, (2014).

[22] M. Shaat, F. F. Mahmoud, X.-L. Gao, A. F. Faheem, Size-dependent bending analysis of Kirchhoff nanoplates based on a modified couple-stress theory including surface effects. Int. J. of Mech. Sci., (2013), http://dx.doi.org/10.1016/j.ijmecsci.2013.11.022i.

[23] A. Arani Ghorbanpour, M. Abdollahian and H. M. Jalaei, Vibration of bioliquid-filled microtubules embedded in cytoplasm including surface effects using modified couple stress theory. J. of Theoret. Biology, 367, 29-38, (2015).

[24] Yong-Gang Wang, Wen-Hui Lin, Ning Liu, Nonlinear bending and post-buckling of extensible microscale beams based on modified couple stress theory. App. Math. Model.39 117-127, (2015).

[25] Ia. S. Podstrigach, Differential equations of the problem of thermodiffusion in isotropic deformed solid bodies. Dop. Akad. Nauk Ukr. SSR, 169-172, (1961).

[26] W. Nowacki, Dynamical problems of thermo diffusion in solids I. Bull Acad. Pol. Sci. Ser. Sci, Tech., 22, 5564, (1974a).

[27] W. Nowacki, Dynamical problems of thermo diffusion in solids II. Bull Acad. Pol. Sci. Ser. Sci, Tech., 22, 129-135, (1974b).

[28] W. Nowacki, Dynamical problems of thermo diffusion in solids III. Bull Acad. Pol. Sci. Ser. Sci, Tech, 22, 257-266, (1974c).

[29] W. Nowacki, Dynamical problems of thermo diffusion in solids. Engg. Frac. Mech., 8, 261-266, (1976).

[30] H.H. Sherief, H. Saleh, F. Hamza, The theory of generalized thermoelastic diffusion. Int. J. Engg. Sci., 42, 591-608, (2004).

[31] H.H. Sherief, H. Saleh, A half-space problem in the theory of generalized thermoelastic diffusion. Int. J. of solid and structures,42, 4484-4493, (2005).

[32] R. Kumar, T. Kansal, Propagation of Lamb waves in transversely isotropic thermoelastic diffusion plate. Int. J. Solid Struc.,45, 5890-5913, (2008).

[33] L. Knopoff, The interaction between elastic wave motion and a magnetic field in electrical conductors. J. Geophys. Res. 60, 441-456, (1955).

[34] P. Chadwick, Ninth Int. Congr. Appl. Mech. 7, 143, (1957).

[35] S. Kaliski, J. Petykiewicz, Equation of motion coupled with the field of temperature in a magnetic field involving mechanical and electrical relaxation for anisotropic bodies. Proc. Vibr. Probl. 4, 1, (1959).

[36] M. Zakaria, Effects of Hall Current and Rotation on Magneto Micropolar Generalized Thermoelasticity due to Ramp-Type Heating. International Journal of Electromagnetics and Applications, 2, 24-32, (2012).

[37] M. Zakaria, Effect of Hall current on generalized magnetothermoelasticity micropolar solid subjected to ramp-type heating. Int. Appl. Mech., 50, 130-144, (2014).

[38] G. Honig, U. Hirdes, A method for the numerical inversion of the Laplace transforms. J. Comput. Appl. Math. 10(1), 113132, (1984).

[39] W.H. Press, S.A. Teukolsky, W.T. Vellerling, B.P. Flannery, Numerical recipes (Cambridge: University Press), (1986). 

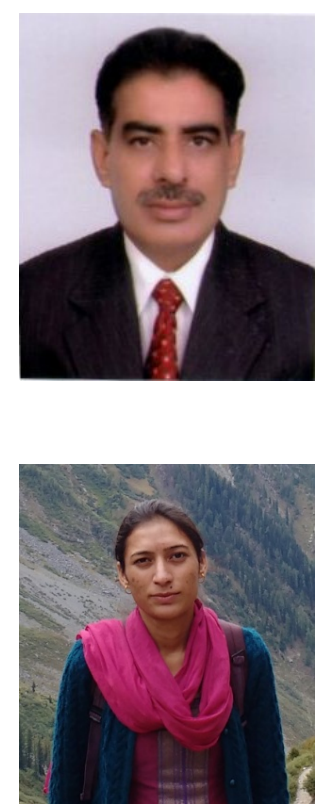

Rajneesh Kumar is a professor at Kurukshetra University Kurukshetra (Haryana, India). He received his PhD in Applied Mathematics from Guru Nanak Dev University, Amritsar (Punjab, India) in 1986. His area of interests include Continuum Mechanics (Micropolar elasticity, thermoelasticity, poroelasticity, magnetoelasticity, micropolar porous couple stress theory, viscoelasticity, double porosity, modified couple stress theory. He has published 448 publications in international journals.

Shaloo Devi is a research scholar at Himachal Pradesh University, Shimla, (Shimla, India). She received her MSc and M. Phil. in Mathematics from Himachal Pradesh University, Shimla (Shimla, India). She has published four research articles and presented three papers at international conferences 